\title{
Seminarios activos: Un recurso para motivar al alumnado universitario al estudio de la Contabilidad
}

\section{Active seminars: A pedagogical resource to motivate students}

Ma Mar Camacho Miñano (marcamacho@ccee.ucm.es)

Elena Urquía Grande (eurquiag@ccee.ucm.es)

David Pascual Ezama (david.pascual@ccee.ucm.es)

Universidad Complutense de Madrid (España)

http://dx.doi.org/10.12795/EDUCADE.2012.i03.06

\begin{abstract}
RESUMEN: La Facultad de Ciencias Económicas y Empresariales de la Universidad Complutense de Madrid, para llevar a cabo la adaptación al Espacio Europeo de Educación Superior, además de rediseñar los planes de estudio ha reestructurado la docencia añadiendo una hora adicional para los seminarios. En la asignatura de contabilidad se han planteado y realizado los denominados "seminarios activos". El objetivo de los mismos es doble: por un lado, que el profesor pueda aplicar una metodología de enseñanza activa y, por otro, integrar los contenidos teóricos del programa a la práctica de la realidad empresarial. Además la enseñanza impartida en los seminarios se ha apoyado en un entorno virtual de aprendizaje.
\end{abstract}

El objetivo de este trabajo es presentar un nuevo recurso para la docencia: los "seminarios activos" a través de la experiencia docente de tres profesores de la asignatura de Contabilidad Financiera de primer curso en los Grados de Economía y de ADE para los seminarios. Posteriormente, se ha realizado una encuesta a los alumnos para la medir su percepción y motivación en estos seminarios.

Los resultados obtenidos de los seminarios activos se pueden resumir en un aprendizaje más profundo de la asignatura y una mayor motivación hacia los contenidos teóricos. No obstante, el alumno ha percibido una carga de trabajo mayor en esta asignatura que en el resto.

PALABRAS ClAVE: Enseñanza activa, Seminarios activos, Entorno virtual de aprendizaje, Contabilidad Financiera, Motivación.

ABSTRACT: The Faculty of Economics and Business Administration from the Complutense University of Madrid in order to adapt to the European Higher Education Area (EHEA) has redesigned the study plan and teaching has been restructured adding an extra hour for seminars. For the accounting subject, active seminars have been proposed. The aim of these seminars is twofold: first, for the teacher to implement an active teaching methodology and, second, to integrate the theoretical content of the subject to the real world business. Additionally teaching has been supported in a virtual learning environment.

This paper presents the experience of three teachers teaching the subject of Financial Accounting in the first-year of the Economics and Business Administration Degrees for the seminars. A survey has been designed to measure the students' perception and motivation towards these seminars.

1 Una versión reducida de este trabajo se ha presentado a la VII Jornada de Campus Virtual de la UCM.

Experiencia docente. Recibido: 26-02-11 - Versiones revisadas: 30-03-11, Aceptado: 10-07-11

Licencia Creative Commons BY NC ND · 2012 · Asociación Española de Contabilidad y Administración de Empresas - AECA 
The results of the active seminars can be summarized in a deeper understanding of the subject and greater motivation to the theoretical concepts. However, the student has received an increased workload in this subject comparing to the others.

KEYWORDS: Active learning, Active seminars, e-learning platform, Financial Accounting, Motivation.

\section{INTRODUCCIÓN}

La educación superior universitaria constituye un valor esencial en el desarrollo cultural, económico y político de un país. En múltiples ocasiones se utilizan indicadores relativos a la universidad: gasto destinado en educación superior, inversión en investigación, número de investigadores, trabajadores con titulación universitaria... para evaluar la riqueza de un país y promover la igualdad de oportunidades en la sociedad (Hanushek, 1998). El principal problema reside en evaluar el impacto de esta docencia en los resultados académicos y profesionales de los estudiantes y, por ende, si son efectivos los recursos destinados a la educación (Levin, 1996).

El nuevo entorno universitario del siglo XXI requiere un esfuerzo pedagógico y docente importante para adaptarse al Espacio Europeo de Educación Superior (EEES). Durante el curso académico 2009/2010 se han implantado las nuevas titulaciones denominadas Grados en la Universidad Complutense de Madrid (UCM). Los retos que conlleva esta adaptación para los docentes de la materia de Contabilidad son los siguientes: aplicar una metodología docente activa para adaptarse al EEES, ajustar la enseñanza de la contabilidad a la realidad empresarial con el apoyo en las Tecnologías de la Información y la Comunicación (TIC) y finalmente, formar a futuros profesionales con el perfil que demanda el mercado laboral.

Para llevar a cabo esta adaptación, la Facultad de Ciencias Económicas y Empresariales de la UCM, además de rediseñar los planes de estudio, ha reestructurado la docencia presencial en clases de tres horas con grupos de 60 alumnos, repartidas en dos sesiones de 90 minutos cada una de ellas, y se ha añadido una "cuarta hora" para los denominados "seminarios". La Facultad ha reorganizado sus recursos disponibles (laboratorios de informática, clases de dimensiones más pequeñas, horarios, etc.) destacando el rediseño de los seminarios ya que el grupo inicial de alumnos se ha subdividido en varios más pequeños para estos seminarios. Además, se han establecido horarios de tutorías personalizadas para cada asignatura. Los autores son profesores con experiencia en grupos pilotos y han diseñado unos seminarios que han denominado "seminarios activos". La filosofía de estos seminarios es poner en práctica la metodología docente activa del EEES en la materia de contabilidad financiera a nivel básico. Estos seminarios tienen una doble finalidad: que el profesor pueda aplicar una metodología de enseñanza activa basada en el constructivismo de Vygotsky, donde el alumno "aprenda a aprender" y, además, integrar los contenidos teóricos de la materia a los aspectos prácticos de la realidad de las empresas. En cuanto a las herramientas de apoyo en las TIC utilizadas por el profesor la docencia presencial con un entorno virtual de enseñanza-aprendizaje (EVEA) denominado Campus Virtual en la UCM ha tenido un papel esencial.

En este trabajo se presenta un nuevo recurso docente que se ha denominado "seminarios activos", mediante la experiencia docente de tres profesores de la asignatura de Contabilidad Financiera de primer curso en los Grados de Economía (GECO) y de Administración y Dirección de Empresas (GADE) para los seminarios. Se diseñaron unos "seminarios activos" en los que se han realizado ejercicios exclusivamente enfocados al mundo empresarial con la finalidad última de mejorar el 
aprendizaje del alumno, aumentar su motivación en el estudio y profundizar en la materia de contabilidad. Los profesores han utilizado el CV como apoyo a una enseñanza interactiva, dinámica y en la que el alumno tuviera el papel central en su aprendizaje.

Este artículo se estructura en los siguientes apartados: en primer lugar, hemos realizado una revisión de la literatura en base a dos líneas de investigación: comparativa de las características de la metodología activa frente a la tradicional y el impacto de esta metodología en la motivación y el tipo de aprendizaje del alumno. A continuación se describen en qué han consistido los seminarios activos, se analiza la encuesta y valoración que han realizado los alumnos. Para finalizar se presentan, de manera resumida, las conclusiones más significativas relativas a la implantación de seminarios activos en la materia de contabilidad financiera. Resulta interesante destacar que los alumnos están muy satisfechos con los seminarios siendo los mejor valorados aquellos que son más activos y requieren más apoyo de las TIC.

Este artículo contribuye a la literatura sobre EEES ya que se plantea un nuevo recurso en la docencia universitaria, los seminarios activos, y se contrastan con la opinión de los alumnos. Además, se comparte esta experiencia con otros docentes para su posible implantación, intentando relacionar la teoría contable con la práctica real de las empresas y motivando al alumno al estudio de la asignatura desde un enfoque utilitarista de la contabilidad financiera.

\section{ESTADO DE LA CUESTIÓN}

La formación universitaria constituye un valor esencial en el desarrollo de un país y, a menudo, se utilizan indicadores relativos a la universidad para evaluar y promover la igualdad de oportunidades en la sociedad. El principal problema reside en evaluar el impacto de esta docencia en los resultados académicos y profesionales del alumnado universitario. Entre los factores que condicionan dichos resultados, analizados desde el punto de vista intrínseco (factores internos al alumno) y extrínseco (factores externos al alumno), destacamos los siguientes:

a) la capacidad cognitiva y madurez del alumno

b) los condicionantes socioeconómicos del entorno (renta familiar, nivel cultural de los progenitores, de los amigos ...)

c) la metodología docente y

d) la motivación del alumnado como actitud positiva hacia el aprendizaje

Estos dos últimos apartados son los que centran nuestro estudio. Mucho se ha escrito sobre las ventajas y desventajas de la metodología docente que fundamenta el EEES (entre otros: García Manjón, 2009; Reyes García, 2007) que sin lugar a dudas se ha convertido en un motor del cambio universitario hacia la mejora de la calidad de la docencia. En los siguientes epígrafes analizaremos la literatura relativa a la diferenciación entre la metodología tradicional con la nueva metodología activa y, a continuación, la interrelación entre la motivación y el tipo de aprendizaje que el alumno adquiere con esta metodología.

\section{1 .la metodología de enseÑanza ACtiva y el APRendizAJE del alumno}

Muchos son los autores que han investigado cómo la metodología de enseñanza del profesor influye en el aprendizaje de los alumnos. Mientras que la definición de una metodología de enseñanza tradicional se centra en el papel del profesor como 
transmisor de contenidos, la metodología activa de enseñanza tiene varios itinerarios y su definición resulta compleja.

En la hora adicional de seminario propuesta por el Decanato de la Facultad se podría haber seguido la metodología docente tradicional. Sin embargo, en la metodología tradicional fundamentada en la clase magistral -método expositivo-, la participación del alumno es escasa y tampoco existe apoyo en las TIC (Wooten, 1998). El aprendizaje tradicional se basa en una mera reproducción de los contenidos previamente expuestos por el profesor y que son evaluados en un único examen final. Esta clase de aprendizaje "tipo fax" (se reproducen los contenidos tal y como lo expone el profesor, sin análisis ni evaluación crítica) se evalúa midiendo la memorización de los contenidos desarrollados por el profesor con una exposición de los mismos en el corto plazo, consiguiendo una aproximación superficial de la asignatura (lo que se denomina "surface learning") (Biggs,2006; Marton y Saljö, 1976; Piaget, 1954). Además la motivación del alumno es muy complicada de conseguir con la metodología docente tradicional (Alonso Tapia (1997)). Por todo ello, un cambio de método se haya justificado, más aún, debido al avance de la sociedad que pone a nuestra disposición herramientas, como los medios informáticos, que facilitan el aprendizaje interactivo. Además, las empresas demandan profesionales capaces de adaptarse al entorno, con capacidad de análisis crítico y motivación para seguir aprendiendo.

En contraposición a la metodología tradicional, la metodología de enseñanza activa se fundamenta en el aprendizaje del estudiante y este es el centro de los denominados "seminarios activos". El profesor pasa a ser un modulador, un intermediario entre los alumnos y los contenidos requeridos. El rol del profesor es ayudarles a adquirir conocimientos, es decir, ayudar a aprender (Marzano, 1991). Así, el alumno tiene un papel autónomo en su aprendizaje, que incluye la selección, organización, elaboración e integración de los contenidos, además de su aplicación a los nuevos escenarios propuestos. De este modo, el aprendizaje adquirido será significativo o "deep learning" (Marton y Sajlö, 1976), es decir, relevante y duradero.

En relación con las materias analizadas, la experiencia de investigación en docencia se ha desarrollado en múltiples campos desde los años sesenta y, en consecuencia, con mucha más profundidad que en la materia contable; destacando las áreas de medicina (Martin et al., 2007; Gordon et al., 2006; Lycke et al. (2006), Gurpinar et al., 2005; Uhari et al., 2003) e ingeniería (Bunting y Cheville, 2009; Holbert y Karady, 2009); Savander-Ranne et al., 2008; Tovia y Liu, 2007; Brown, 2005; Terenzini et al., 2001; Yuretich et al., 2001). Sin embargo, desde hace un tiempo también se vienen realizando estudios que corresponden al área de la Contabilidad Financiera (Camacho, 2009; Halabi et al., 2005; Arquero et al., 2004), de Auditoría (Still y Clayton, 2004), de Contabilidad de Gestión (Urquía et al., 2009; Nouri y Shahid, 2005), de Contabilidad Analítica (Clinton y Kohlmayer, 2005) o incluso en Contabilidad Fiscal (Manly et al., 2005). Como se observa, en materias técnicas ha habido un mayor interés por la investigación docente.

A diferencia de la metodología tradicional que se fundamenta en la clase magistral, la metodología activa incluye diferentes herramientas para conseguir la interacción con el alumno. Merece la pena destacar algunas de ellas: mediante transparencias en Power Point (Nouri y Shahid, 2005), "quizzes" en grupos al final de la clase (Clinton y Kohlmeyer, 2005), ejercicios interactivos donde los alumnos van aprendiendo por descubrimiento (Halabi et al., 2005), tutorías personalizadas (Dillard-Eggers y Wooten, 2003), mandos interactivos (Camacho, 2009; Uhari et al., 2003), casos reales de empresas (Arquero et al. (2004)), simulaciones (Urquía et al.; 2009; Potter y Johnston, 2006) y el aprendizaje basado en problemas (ABP) (Heagy y Lehman, 2005). Cuando estas herramientas de aprendizaje activo además se combinan con una forma de agrupar a los alumnos para que trabajen en equipo, dependiendo de cómo sea el 
trabajo entre los alumnos y cómo sea la evaluación (Prince, 2004, Prince y Felder, 2006), este aprendizaje se denomina cooperativo (Hwang et al., 2005; Felder, 1995) o colaborativo (Walker et al., 2008). Adicionalmente, las empresas demandan cada vez más profesionales que sepan trabajar en equipo, resolver problemas y tener una actitud crítica.

Además, independientemente de la herramienta utilizada, si es activa conllevará siempre un apoyo en las TIC (entre otros: Potter y Johnston, 2006; Bryant y Hunton, 2000). Las TIC aplicadas a la docencia son consideradas como el motor del cambio en el aprendizaje de los alumnos siempre y cuando exista una flexibilidad del método docente y una motivación a su uso (Salinas, 2004). Desde los años 90 los investigadores, sobre todo en EE.UU., han analizado el efecto del apoyo de las TIC en la enseñanza y en el aprendizaje de los alumnos (Holbert y Karadi, 2009; Yuretich et al., 2001; Uhari et al., 2003; Bautista, 1994).

En este punto es donde nos planteamos la siguiente pregunta de investigación:

PI 1: ¿̇Consigue la metodología docente activa de los recursos diseñados y aplicados en los seminarios de Contabilidad Financiera un aprendizaje más profundo de la materia?

\subsection{IMPACTO dE LA METOdOLOGía DOCENTE ACTIVA EN LA MOTIVACIÓN DEL ALUMNO}

Muchos de los autores mencionados en el epígrafe anterior analizan el impacto, con resultados positivos concluyentes, de la aplicación de una metodología docente activa en el aprendizaje de los alumnos combinado con el efecto de haber conseguido una mayor motivación. Del Favero et al., (2007) y Wooten, (1998), entre otros, realizan experimentos para sus asignaturas y diseñan diferentes modelos con ecuaciones estructurales donde se relacionan variables extrínsecas del alumno (familia, ambiente de estudio...) con variables intrínsecas (aptitud, esfuerzo, motivación...) para llegar a unas relaciones causa-efecto donde la motivación es una de las variables causa del aprendizaje profundo y duradero del alumno.

Hay autores que demuestran directamente que aquellos alumnos con mayor motivación inducida por el aprendizaje a través de la metodología docente activa han sido los que mejores resultados académicos han obtenido. Dependiendo de las herramientas utilizadas en esta metodología activa, si ha sido aplicada a través del método del caso (Ochsendorf et al., 2006), o con el aprendizaje basado en problemas (ABP) (Nalesnik et al., 2004) o mediante simulaciones (Seybert y Barton, 2007) u otro tipo de herramientas (Aston-Brown et al., 2009; Fakhry et al., 2007). En la misma línea los trabajos en equipo, ya sean colaborativos o cooperativos, consiguen también una mejora de la motivación y aprendizaje (Cheng et al., 2009).

Sin embargo, otros autores llegan a resultados no concluyentes puesto que aunque demuestran que la metodología docente activa ha aumentado la motivación de los alumnos, esto no es suficiente para aumentar y robustecer su aprendizaje (LópezFernández y Rodríguez-lllera, 2009; Maag, 2004). Finalmente hay autores que no llegan a demostrar que la metodología docente activa mejora ni la motivación de los alumnos ni el aprendizaje (Tam et al., 2009; Engum et al., 2003; Parkinson y Ekachai, 2002) comparado con el uso de la metodología docente tradicional.

Desde nuestro punto de vista estamos de acuerdo en considerar al profesor como "motor de impulso" para el aprendizaje del alumno (Bain, 2007). Aunque existe una evidente aversión al cambio en cualquier proceso novedoso, el profesor universitario hoy en día tiene un rol muy importante que desempeñar, utilizando una metodología activa apoyada con las TIC. En este trabajo hemos incidido en la acción combinada de los seminarios activos con el apoyo del Campus Virtual. Los ejercicios con el marco 
teórico, las normas y las preguntas para trabajar en los seminarios se han recogido en la plataforma on-line de la asignatura desde el principio de curso y cada vez que se había terminado el trabajo solicitado por el profesor, tanto en equipo como individual, se debía entregar. La evaluación de los trabajos se ha hecho a través del CV y era enviada en el menor tiempo posible, con la finalidad de que el "feed-back" existiera y fuera eficaz, dada la importancia del mismo en la motivación y el aprendizaje del alumno (Branch, 2002).

En este apartado nos planteamos la siguiente pregunta de investigación:

PI2: ¿Ha aumentado la metodología activa de los recursos diseñados y aplicados en los seminarios la motivación de los alumnos hacia el aprendizaje de la Contabilidad Financiera?

Con este artículo se pretende aportar en el área de la contabilidad financiera nuevos recursos que mejoren la calidad docente de la materia.

\section{METODOLOGÍA EMPÍRICA}

\subsection{MUESTRA}

La muestra ha consistido en los alumnos de cuatro grupos de la asignatura de Contabilidad Financiera. La muestra final se compone de un total de 135 alumnos. De los 135 alumnos 47 eran del Grado de Economía y el resto del Grado de ADE. El $42 \%$ eran hombres con una edad media de 19 años y de los cuales tan sólo había un $9 \%$ de alumnos que cursaban la asignatura por segunda vez.

En todo análisis exploratorio de datos hay un conjunto de datos perdidos por ausencia de información sobre el individuo muestreado con lo que se ha procedido a realizar un test de aleatoriedad para observar si estos datos perdidos se distribuyen aleatoriamente. En este trabajo se ha realizado el test de aleatoriedad de rachas de Wald-Wolfowitz y al observar que tanto el porcentaje de datos perdidos $(2,3 \%$ de la muestra) era pequeño como que dichos datos se comportaban aleatoriamente se procedió a eliminarlos. La técnica de eliminación ha sido de los individuos con datos perdidos en alguna de las variables.

\subsection{INSTRUMENTO}

Este cuestionario se ha diseñado y contrastado con distintos profesores para validar la utilidad del mismo y se ha realizado con la colaboración de los alumnos al final del curso. En un primer apartado se quería evaluar la satisfacción y cobertura de expectativas de los alumnos en sentido general. En un segundo apartado se graduarían los diferentes seminarios en función de la percepción del alumno, su grado de dificultad y su utilidad. En un tercer apartado se les cuestionaría sobre las ventajas e inconvenientes de estos seminarios. Por último en un cuarto apartado se investigaría sobre el grado de satisfacción con el uso del CV como apoyo al aprendizaje y enseñanza de la asignatura (ver Anexo 1). Las variables analizadas se detallan en la Tabla 1.

\section{3 .PROCEDIMIENTO}

En la denominada "cuarta hora" de docencia en la asignatura de "contabilidad financiera" se han desarrollado cinco seminarios prácticos con una doble finalidad: por un lado, motivar al alumno en el aprendizaje y estudio de la materia y, por otro lado, completar las clases teórcas con un acercamiento a la práctica real de las empresas y a sus futuros puestos de trabajo (Tabla 2). 
Todos los seminarios han estado apoyados en el CV utilizando la plataforma Moodle. La estructura que se ha seguido en el diseño de los contenidos, que se iban actualizando para la consulta de los alumnos en todo momento, ha sido dividir en tantos capítulos como se iban a impartir en las clases y, al final, un apartado para los seminarios, que se iban desarrollando en paralelo a la docencia teórico-práctica de las clases. Cada sección denominada "capítulo" tiene una estructura común y ha constado de cuatro apartados: una introducción, la documentación de referencia, unos ejercicios prácticos y unos recursos adicionales. En el apartado de "introducción" se han destacado los objetivos de aprendizaje para el alumno. En el apartado de documentación se han "colgado" la nota técnica, la presentación en PowerPoint que el profesor ha desarrollado en clase y un glosario de los términos contables específicos. En el apartado de "ejercicios" se han añadido los supuestos prácticos que pertenecían a ese marco teórico. Finalmente, en el epígrafe de "recursos" se han insertado unos vínculos con páginas Web interesantes que el alumno debía consultar para complementar el tema, noticias de prensa relacionadas con la materia, además de bibliografía complementaria, con la finalidad de "filtrar" el exceso de información existente en Internet (Lyman y Varian, 2003).

TABLA 1. DESCRIPCIÓN DE LAS VARIABLES UTILIZADAS Y SU TABULACIÓN

\begin{tabular}{|c|c|}
\hline SAT & $\begin{array}{l}\text { Satisfacción de los alumnos sobre los conocimientos adquiridos en los } \\
\text { seminarios. (Ver Anexo. Pregunta 1) }\end{array}$ \\
\hline EXP & $\begin{array}{l}\text { Expectativas cubiertas por parte de los alumnos en relación a lo que } \\
\text { esperaban de los seminarios a priori (Ver Anexo. Pregunta 2) }\end{array}$ \\
\hline VSEMi & $\begin{array}{l}\text { Valoración general de cada uno de los seminarios por parte de los alumnos } \\
\text { (Ver Anexo. Pregunta 3) }\end{array}$ \\
\hline dSEMi & $\begin{array}{l}\text { Valoración de la dificultad encontrada en el desarrollo de cada uno de los } \\
\text { seminarios por parte de los alumnos. (Ver Anexo. Pregunta 4) }\end{array}$ \\
\hline USEMi & $\begin{array}{l}\text { Valoración de la utilidad percibida de cada uno de los seminarios por parte de } \\
\text { los alumnos. (Ver Anexo. Pregunta 5) }\end{array}$ \\
\hline VGSEM & $\begin{array}{l}\text { Valoración global de los seminarios por parte de los alumnos (Ver Anexo. } \\
\text { Pregunta 6). }\end{array}$ \\
\hline ISEM & $\begin{array}{l}\text { Valoración de los inconvenientes de los seminarios percibidos por los alumnos. } \\
\text { (Ver Anexo. Pregunta 7) }\end{array}$ \\
\hline CSEM & $\begin{array}{l}\text { Valoración de los seminarios por parte de los alumnos respecto a otro tipo de } \\
\text { seminarios (Ver Anexo.Pregunta 8) }\end{array}$ \\
\hline uSOCV & Utilización del Campús Virtual por parte de los alumnos. (Ver Anexo. Pregunta 9) \\
\hline SATCV & $\begin{array}{l}\text { Valoración de la satisfacción del uso del Campús Virtual en los seminarios por } \\
\text { parte de los alumnos. (Ver Anexo. Pregunta 10). }\end{array}$ \\
\hline
\end{tabular}




\section{TABLA 2. DESCRIPCIÓN DE LOS SEMINARIOS}

\begin{tabular}{|c|l|}
\hline $\begin{array}{c}\text { Seminario 1: } \\
\text { CNMV }\end{array}$ & $\begin{array}{l}\text { Búsqueda de información financiero-contable en páginas Web oficiales } \\
\text { (Comisión Nacional del Mercado de Valores (CNMV), Registro Mercantil } \\
\text { (RM)...etc.) }\end{array}$ \\
\hline $\begin{array}{c}\text { Seminario 2: } \\
\text { SABI }\end{array}$ & $\begin{array}{l}\text { Búsqueda de información de empresas reales en la base de datos de } \\
\text { empresas SABI }\end{array}$ \\
\hline $\begin{array}{c}\text { Seminario 3: } \\
\text { MEMORIA }\end{array}$ & $\begin{array}{l}\text { Lectura e interpretación de la Memoria de las Cuentas Anuales de una } \\
\text { empresa real, información pública que utilizarán en su vida laboral de } \\
\text { manera directa o indirecta }\end{array}$ \\
\hline Seminario 4: & $\begin{array}{l}\text { Introducción de hechos contables en el programa informático A3CON, } \\
\text { incluyendo un supuesto con facturas reales para que el alumno realice } \\
\text { la llevanza de la contabilidad como en cualquier empresa real }\end{array}$ \\
\hline $\begin{array}{c}\text { Seminario 5: } \\
\text { PRESENTACION }\end{array}$ & $\begin{array}{l}\text { Presentación oral de la Memoria de una empresa real y un breve } \\
\text { análisis de su lectura }\end{array}$ \\
\hline
\end{tabular}

La dinámica de los seminarios se dividía en dos partes: un trabajo individual del alumno en el aula de informática, teniendo que rellenar un cuestionario, preguntas breves o tipo test, y un trabajo fuera del aula, teniendo que realizar un trabajo en grupo y enviando el ejercicio a través del CV. En la sección de los seminarios del CV se han diferenciado también tres apartados: -el marco teórico del seminario, -una nota técnica con las instrucciones del ejercicio práctico que tenían que realizar los alumnos y -un vínculo para que los alumnos enviasen por el CV el trabajo asignado. El profesor calificó estos trabajos siempre en un plazo inferior a una semana a través del CV para que el alumno tuviera el feedback en el corto plazo como elemento de motivación hacia la mejora de su aprendizaje.

\section{RESULTADOS Y DISCUSIÓN}

En primer lugar, hay que destacar que un $79 \%$ de los alumnos está satisfecho con lo aprendido en los seminarios y un $72 \%$ del alumnado ha cubierto sus expectativas iniciales para el primer curso de la materia. Además, hay que resaltar que el $95 \%$ de los alumnos utilizaron el CV para el desarrollo de los seminarios, valorando muy positivamente el uso de esta herramienta virtual (ver Gráfico 1).

\section{Gráfico 1. Valoración de la satisfacción del uso del CV}

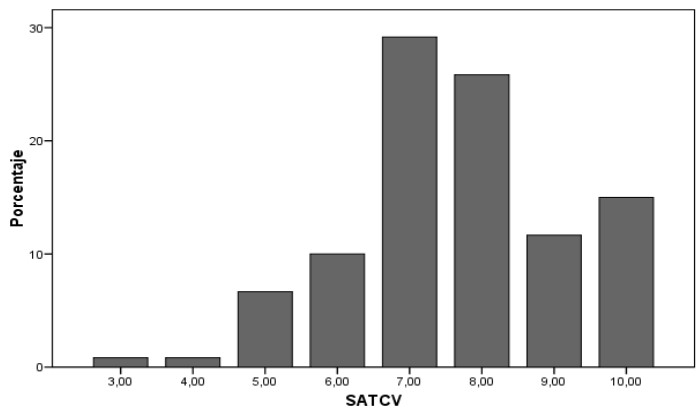

\footnotetext{
2 Sistema de Análisis de Balances Ibéricos
} 
Con relación a la valoración global de los seminarios (ver Tabla 2), la mayoría ha considerado que "en los seminarios se aprende a conocer la utilidad de los contenidos teóricos combinados con un enfoque dinámico y real" aunque uno de los principales inconvenientes (ver Tabla 3) es que "la carga de trabajo de los seminarios es muy elevada" incluso si se compara con la exigida en otras asignaturas. De hecho, el $80 \%$ de los alumnos ha percibido que los seminarios se complementaban con los contenidos teórico-prácticos de las clases. Sobre el total de matriculados, las actividades de los seminarios han sido realizadas por un $65 \%$ del total del alumnado matriculado, de los cuales más del $70 \%$ han aprobado los seminarios.

TABLA 2. VAloración global de los Seminarios

\begin{tabular}{|l|l|c|c|c|c|}
\hline \multicolumn{2}{|c|}{} & Frecuencia & Porcentaje & $\begin{array}{c}\text { Porcentaje } \\
\text { válido }\end{array}$ & $\begin{array}{c}\text { Porcentaje } \\
\text { acumulado }\end{array}$ \\
\hline \multirow{4}{*}{ Válidos } & ENFOQUE PRACTICO & 44 & 33,1 & 33,8 & 33,8 \\
\cline { 2 - 6 } & ENFOQUE TEORICO & 59 & 44,4 & 45,4 & 79,2 \\
\cline { 2 - 6 } & INCREMENTO CARGA & 24 & 18,0 & 18,5 & 97,7 \\
\cline { 2 - 6 } & NADA INTERESANTE & 3 & 2,3 & 2,3 & 100,0 \\
\cline { 2 - 6 } & Total & 130 & 97,7 & 100,0 & \\
\hline Perdidos & Sistema & 3 & 2,3 & & \\
\hline Total & 133 & 100,0 & & \\
\hline
\end{tabular}

TABLA 3.- INCONVENIENTES DE LOS SEMINARIOS

\begin{tabular}{|c|c|c|c|c|c|}
\hline & & Frecuencia & Porcentaje & $\begin{array}{c}\text { Porcentaje } \\
\text { válido }\end{array}$ & $\begin{array}{l}\text { Porcentaje } \\
\text { acumulado }\end{array}$ \\
\hline \multirow[t]{5}{*}{ Válidos } & CARGA ELEVADA & 54 & 40,6 & 43,2 & 43,2 \\
\hline & FALTA COORDINACION & 26 & 19,5 & 20,8 & 64,0 \\
\hline & NO HAY FEEDBACK & 10 & 7,5 & 8,0 & 72,0 \\
\hline & NINGUNO & 35 & 26,3 & 28,0 & 100,0 \\
\hline & Total & 125 & 94,0 & 100,0 & \\
\hline $\begin{array}{c}\text { Perdid } \\
\text { os }\end{array}$ & Sistema & 8 & 6,0 & & \\
\hline \multicolumn{2}{|r|}{ Total } & 133 & 100,0 & & \\
\hline
\end{tabular}

Valorando cada uno de los seminarios en particular (ver Gráfico 2), el número 2 de búsqueda de información en $S A B I$ ha sido el mejor valorado seguido del seminario 1 , búsqueda de información contable en páginas web oficiales. Cabe resaltar que las diferencias en las puntuaciones de valoración del seminario 2 son estadísticamente significativas con el resto. Respecto a la dificultad, el seminario 4 de uso del programa informático $\mathrm{A} 3 \mathrm{CON}$ ha resultado más complejo para los alumnos y las diferencias han sido estadísticamente significativas con el resto. Con relación a la utilidad, el mencionado seminario ha sido considerado significativamente más útil que el resto de los seminarios, a excepción del seminario 2, SABI, en el que las diferencias no son estadísticamente significativas.

Por otra parte, existe una relación estadística significativa entre la valoración de cada seminario y su percepción de utilidad salvo en el seminario 1. Esto podría justificarse porque en este seminario se trata simplemente de una búsqueda de información en Internet sobre las cuentas anuales de las empresas que, posteriormente, tendrán que analizar en el seminario 3 y presentar oralmente en el seminario 5. Hay que destacar 
también que en ningún seminario existe una relación significativa entre la valoración de los mismos y su dificultad. Podría deberse a que la utilidad prima en la valoración por parte de los alumnos.

\section{GRÁfico 2. VALORACIÓN, DIFICULTAD Y UTILIDAD DE LOS SEMINARIOS}

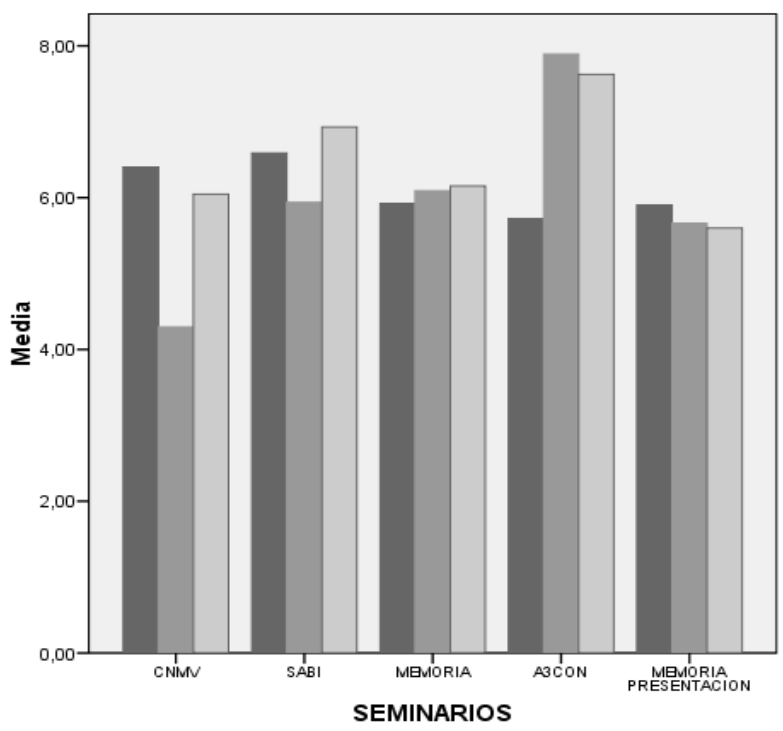

Sin embargo, los seminarios 1 (búsqueda de información en Internet) y 2 (SABI), con menos carga de trabajo y que requieren menos conocimientos previos, han sido los mejor valorados. Se puede concluir que los seminarios activos han acercado la realidad empresarial al alumno y han motivado al estudio de los aspectos teóricos de la materia. Sin embargo, un $40 \%$ de los alumnos ha percibido un exceso de carga de trabajo y de dificultad comparado con otros seminarios de otras asignaturas. También es interesante resaltar que uno de cada cuatro alumnos no encuentra inconvenientes a los seminarios de contabilidad financiera.

Por último, destacar que han aprobado los seminarios en mayor medida que la asignatura final, que es un $55 \%$ de media sobre el total de los presentados. Además, los alumnos que han aprobado la asignatura coinciden con los que han presentado las actividades de los seminarios activos.

\section{CONCLUSIONES.}

A raíz de las preguntas de investigación planteadas y los resultados estadísticos obtenidos hay que destacar que los alumnos están satisfechos con los seminarios "activos", siendo éste el primer año de experiencia docente. Es interesante resaltar que aquellos más activos, donde se entrelazan más TIC, son los mejor valorados por los alumnos y donde han usado más el CV. Además, las calificaciones de estos seminarios son mejores. De este modo, se han vinculado las características particulares de los nuevos alumnos de la universidad, la denominada generación Z, o generación ".neł con sus intereses, en los que predomina el uso de los recursos electrónicos (Zamora, 2009). La metodología docente utilizada en los seminarios activos se vincula con los intereses de los alumnos actuales, y su aprendizaje también. En otras palabras, con los seminarios activos se consigue adaptar la metodología docente a las exigencias del EEES acercando los contenidos teóricos de la materia a la realidad empresarial.

Uno de los resultados más relevantes es que los seminarios que han requerido una profundidad en los conocimientos previos de la materia, son aquellos peor valorados y 
considerados más difíciles. De hecho, una característica particular de la asignatura de contabilidad financiera es la acumulación de contenidos en el desarrollo del programa, es decir, que no se trata de temas independientes sino que están ligados unos con otros. Por ello, la realización de los últimos seminarios ha requerido unos conocimientos teórico-prácticos previos, con una mayor carga de trabajo.

Por otro lado, aquellos seminarios que han utilizado programa informáticos novedosos para los alumnos (SABI y A3CON) han sido valorados como muy útiles, a pesar de su alto grado de dificultad. Este hecho contradice la "cultura-del-poco-esfuerzo" de nuestros jóvenes ya que los alumnos han valorado muy positivamente aquellos seminarios complejos pero útiles para su desarrollo profesional en el futuro.

El objetivo de motivar al alumno al estudio de la contabilidad se ha conseguido porque individualmente han necesitado estudiar la materia teórica para realizar los seminarios. De este modo, constatamos que el alumno motivado mejora sus resultados. Así respondemos a las dos preguntas de investigación planteadas.

Entre las limitaciones del estudio se destacan el tamaño de la muestra pero ha resultado complicado evaluar a los alumnos a final de curso, ya que la asignatura es de segundo cuatrimestre. Por otro lado, el cuestionario se va a rediseñar para futuros estudios en función de la experiencia de este año.

A pesar de que los seminarios activos han acercado la realidad empresarial al alumno ya desde el primer curso de su carrera y han motivado un análisis más detallado de aspectos teóricos, ha habido una percepción por parte del alumno de tener un exceso de carga de trabajo y dificultad con estos seminarios. Los profesores han de reflexionar en cómo hay que reorganizarlos para el próximo curso.

En líneas generales, tratándose de un primer año de implantación de los seminarios activos se considera que los resultados de su evaluación son más que satisfactorios. En un futuro se espera seguir trabajando en esta línea de investigación de enseñanza activa y motivación al aprendizaje significativo, mejorando los contenidos e intentando solucionar los problemas percibidos.

Por último, este trabajo de investigación aporta una mejora objetiva de la docencia universitaria ya que los resultados obtenidos revertirán directamente en el progreso futuro de la asignatura. Sin embargo, en la actualidad, como los docentes están teniendo que diversificar sus esfuerzos entre investigación, docencia y gestión, lo que condiciona su promoción, se produce una dispersión entre tales actividades, que afectan negativamente a la docencia. En este momento, la investigación está mucho más valorada en las acreditaciones docentes y no siempre incide en la materia impartida en clase. En este trabajo pretendemos aunar la investigación con la docencia en aras de la mejora continua de su calidad en las aulas universitarias.

\section{BIBLIOGRAFÍA}

AlONSO TAPIA, J. (1997): Motivar para al aprendizaje. Teoría y estrategias. Barcelona: EDEBÉ.

ARQUERO J.L., JIMÉNEZ, S.M Y JOYCE, J. (2004): Skills development, motivation and learning in financial statement analysis: an evaluation of alternative types of case studies. Accounting Education, 13(2): 191-212.

Aston-Brown, R.E., Branson, B., GADBURY-AMYOT, C.C y BRAY, K.K. (2009): Utilizing Public Health for service learning rotations in dental hygiene: a four year retrospective study. Journal of Dental Education, 73(3): 358-374.

BAIN, K. (2007): Lo que hacen los mejores profesores universitarios. Barcelona: Universitat de Valencia. 
Bautista García-Vera, A. (1994): Las nuevas tecnologías en la capacitación docente. Madrid: Ed. Aprendizaje Visor.

BIGGS, J. (2006): Calidad del aprendizaje universitario. $2^{a}$ edición. Madrid: Narcea, SA de ediciones.

BRANCH, W. (2002): Feedback and reflection: teaching methods for clinical settings. Academic Medicine, 77(12): 1185-1188.

BROWN, N. (2005): Meta programmes for identifying thinking preferences and their impact on Accounting students educational experience. Journal of Accounting Education, 23(4): 232-247.

BRYANT, S.M. y HUNTON, J.E. (2000): The use of technology in the delivery of instruction: Implications for Accounting Educators and Education Researchers. Issues in Accounting Education, 15(1): 129-162.

BUNTING, C. F. Y CHEVILLE, R. A. (2009): VECTOR: a hands-on approach that makes electromagnetics relevant to students. IEEE Transactions on Education, 52(3): 350-359.

CAMACHO MiñANo, M. (2009): Uso de mandos interactivos en la docencia universitaria. Aplicación práctica en la asignatura de Contabilidad Financiera I. Proyecto MATHEMATICAL-LEARNING. Disponible en: http://cimanet.voc.edu/mel/content/view/75/82/lang,spanish/. Comunicación y póster presentados en las $\mathrm{V}$ Jornadas del Campus VirtualUCM.

CHENG, E.K., RHEE, J.A., BAIK, Y.H. y OS, A. (2009): The effect of team based learning in medical ethics education. Medical Teacher, 31 (11): 1013-1017.

CLINTON, B.D. Y KOHLMAYER III, J.M. (2005): The effects of group quizzes on performance and motivation to learn: two experiments in cooperative learning. Journal of Accounting Education, 23: 96-116.

Dillard-EgGers, J. y WOOTEN, T. (2003): The use of peer tutors in Introductory Financial Accounting. Advances in Accounting Education, 5: 55-80.

Del FaVero, L., Boscolo, P., Vidotto, G. y VicentinI, M. (2007): Classroom discussion and individual problem-solving in the teaching of History: Do different instructional approaches affect interest in different ways? Learning and Instruction, 17: 635657

ENGUM, S.A., JefFRIES, P. Y FISHER, L. (2003): Intravenous catheter training system: Computer based education versus traditional learning methods. American Journal of Surgery, 186(1): 67-74.

FAKHRY, A. Y DEHKORDI-VAKIL, F.H. (2007): Video assisted clinical instruction in dentristry (VACID) enhances real time visualisation of clinical procedures. European Journal of Dental Education, 11 (4): 238-244.

FELDER, R.M. (1995): Cooperative learning in a sequence of engineering courses: a success story. Cooperative Learning and College Teaching Newsletter, 5(2): 10-13.

GARCíA MANJÓN, J.V., (COORD.) (2009): Hacia el espacio europeo de educación superior: el reto de la adaptación de la universidad a Bolonia. Oleiros (La Coruña): Netbiblo, D.L.

Gordon, J.A., Shaffer, D. W., Raemer, D. B., Pawlowski, J., Hurford, W.E. y CoOper, J. B. (2006): A randomized controlled trial of simulation-based teaching versus traditional instruction in medicine: a pilot study among clinical medical students. Advances in Health Sciences Education, 11: 33-39.

GurPINAR, E., MUSAL, B., AKSAKOGLU, G. Y UCKU, R. (2005): Comparison of knowledge scores of medical students in problem-based learning and traditional curriculum on public health topics. BMC Medical Education, 5(7): 1-8.

HALABI, A.K., TUOVINEN, J.E. Y FARLEY, A.A. (2005): Empirical evidence on the relative efficiency of worked examples versus problem-solving exercises in Accounting Principles instruction. Issues in Accounting Education, 20(1): 21-32. 
HANUSHEK, E. (1998): Conclusions and controversies about the effectiveness of school resources. Economic Policy Review, March: 11-27.

HeAGY, C.D. Y LEHMANN, C.M. (2005): Is PBL an improved delivery method for the Accounting curriculum? Advances in Accounting Education, 7: 225-251.

HOLBERT, K.E. Y KARADY, G.G. (2009): Strategies, Challenges and prospects for active learning in the computer-based classroom, IIIE Transactions on Education, 52 (1): 31-38.

HWANG, N.C.R., LUI, G. Y WU TONG, Y.J. (2005): An empirical test of cooperative learning in a passive learning environment. Issues in Accounting Education, May: 151-165.

LEVIN, H. (1996): Aumentando la productividad educativa. Economía de la Educación. Temas de Estudio e Investigación Estudios y Documentos, (22), I. Servicio de Publicaciones del Gobierno Vasco.

LÓPEZ-FERNÁNDEZ, O. Y RODRíGUEZ-ILLERA, J.L. (2009): Investigating university's students adaptation to a digital learner course portfolio. Computers and Education, 52(3): 608-616.

LYCKE, K.H., GROTTUM, P. Y STROMSO, H.I. (2006): Student learning strategies, mental models and learning outcomes in problem-based and traditional curricula in medicine. Medical Teacher, 28(8): 717-722.

LYMAN, P. Y VARIAN, H.R. (2003): How much information 2003?. Disponible en: http://www.sims.berkeley.edu/how-much-info-2003. (Acceso el 2 de septiembre de 2010)

MAAG, M (2004): The effectiveness of an interactive multimedia learning tool on nursing students'math knowledge and self efficacy. Cin Computers Informatics Nursing, 22(1): 26-33.

MANLY, T. S., WALSH, R. J. Y MCKNIGHT, C. A. (2005): Measurement of earnings, taxes and ecommerce: The case of Calypso Communication. Global Perspectives on Accounting Education, 2: 1-10.

MARTIN, T.; RIVALE, S.D. y DILLER, K.R. (2007): Comparison of student learning in challengebased and traditional instruction in Biomedical Engineering. Annals of Biomedical Engineering, 35(8): 312-323.

MARTON, F. Y SALJÖ, R. (1976): On qualitative differences in learning: I, outcome and process. British Journal of Educational Psychology, 46(1): 4-1 1.

MARZANO, R. J. (1991): Creating an educational paradigm centered on learning through teacher-directed, naturalistic inquiry. En L. Idol y B. F. Jones (Eds.), Educational values and cognitive instruction. Implication for reform (411-442). Hillsdale: Erlbaum.

NAlesnik, S.W., Heaton, J.O., Olsen, C.H., HaffNer, W.H.J. y ZAHN, C.M. (2004): Incorporating problem based learning into obstetrics/gynecology clerkship: Impact on student satisfaction and grades. American Journal of Obstretics and Gynecology, 190(5): 1375-1381.

NouRI, H. Y SHAHID, A. (2005): The effect of Power Point presentations on student learning and attitudes. Global Perspectives on Accounting Education, 2: 53-73.

OCHSENDORF, F.R., BOEHNCKE, W.H., SOMMERLAD, M. Y KAUfMANN, R. (2006): Interactive large group teaching in a dermatology course. Medical Teacher, 28(8): 697-701.

PARKINSON, M.G Y EKACHAI, D. (2002): The Socratic method in the introductory PR course: an alternative pedagogy. Public Relations Review, 28(2): 167-174.

PIAGet, J. (1954): The Construction of Reality in the Child". New York: Basic Books. http://books.google.es/books?hl=es\&lr=\&id=hK37xrpadlkC\&oi=fnd\&pg=PA3\&d $\mathrm{q}=$ The+Construction+of+Reality+in+the+Child\&ots=ye6HfSJDeW\&sig=Rj4GV3H -5OZURhJ53O2c4LVszl\#v=onepage\&q\&f=false. (Acceso 6 de septiembre de 2010).

POTTER, B.N. Y JOHNSTON, C.G. (2006): The effect of interactive on-line learning systems on student learning outcomes in accounting. Journal of Accounting Education, 24: 16-34. 
PRINCE, M.J. y FELDER, R.M (2006): Inductive teaching and learning methods: definitions, comparisons and research bases. Journal of Engineering Education, April: 1-16.

PRINCE, M.J. (2004): Does active learning work? A review of the research. Journal of Engineering Education, July: 1-9.

ReYES GARCÍA, C.I. (2007): El espacio europeo de educación superior: algunas cuestiones clave. Las Palmas de Gran Canaria: Universidad.

SALINAS, J. (2004): Innovación docente y uso de las TIC en la enseñanza universitaria. Revista Universidad y Sociedad del Conocimiento, 1 (1): 1-16.

SAVANDER-RANNe, C., LUDEN, O.P. y Kolarl, S. (2008): An alternative teaching method for electrical engineering courses. IEEE Transactions on Education, 51 (4): 423-431.

SEYBERT, A.L. y BARTON, C.M. (2007): Simulation based learning to teach blood pressure assessment to doctor of pharmacy students. American Journal of Pharmaceutical Education, 71 (3): 1-6.

STILL, K. Y CLAYTON, P. R. (2004): Utilizing service-learning in accounting programs. Issues in Accounting Education, 19(4): 469-486.

TAM, M.D.B.S., HART, A.R., WILLIAMS, S., HEYLINGS, D. y LEINSTER, S. (2009): Is learning anatomy facilitated by computer aided learning? A review of the literature. Medical Teacher, 31 (9): 393-396.

Terenzinl, P.T., Cabrera, A.F., ColbeCk, C.L., Parente, J.M. y Bjorklund, S. A. (2001): Collaborative Learning vs Lecture/Discussion: Students' reported learning gains. Journal of Engineering Education, January: 123-130.

TOVIA, F. Y LIU, Y. (2007): Students evaluating significant factors on retention: a statistical analysis. International Conference on Engineering Education- ICEE, September 3-7. Coimbra Portugal.

UhARI, M.; RENKO, M. y SOINI, H. (2003): Experiences of using an interactive audience response system in lectures. BMC Medical Education, 3(12): 1-6.

URquía Grande, E., Muñoz Colomina, C.I. y Cano Montero, E. I. (2009): La simulación del cuadro de mando integral. Una herramienta de aprendizaje en la materia de contabilidad de gestión. Documento de trabajo n 465/2009. Fundación de las Cajas de Ahorro.

WAlker, J.D., Cotner, S. H., BAepler, P. M. y DeCKer, M.D. (2008): A delicate balance: integrating active learning into a large lecture course. The American Society for Cell Biology, 7: 361-367.

WOOTEN, T. C. (1998): Factors influencing student learning in introductory Accounting Classes: a comparison of traditional and non-traditional students. Issues in Accounting Education, 13(2): 357-373.

YURETICH, R.F., KHAN, S.A., LECKIE, R.M. Y CLEMENT, J.J. (2001): Active-Learning methods to improve student performance and scientific interest in a large introductory Oceanography course. Journal of Geoscience Education, 49(2): 111-119.

ZAMORA CARRILLO, E. (2009): Nuevos modelos educativos para nuevas generaciones: La generación net. http://portal.educar.org/edgar-zamora-carrillo/nuevosmodelos-educativos-para-nuevas-generaciones-la-generacionnet\#comment-15719 (Acceso 1 septiembre 2010). 


\section{ANEXO 1: ENCUESTA REALIZADA A LOS ALUMNOS}

ENCUESTA SOBRE LOS SEMINARIOS

\section{DE CONTABILIDAD FINANCIERA}

1.- ¿̇Está satisfecho con el contenido de lo aprendido en los seminarios? (Marque con una X)

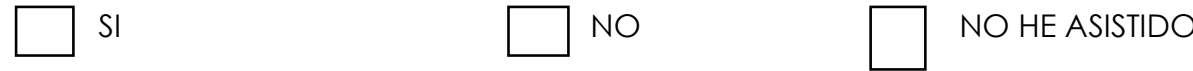

2.- Los seminarios de contabilidad financiera zhan cubierto sus expectativas iniciales durante su primer año de Grado?

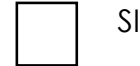

SI

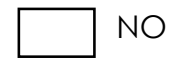

3.- Del 1 al 10 ¿qué nota le pondría a cada seminario en función de su percepción personale (1 min., 10 máx.).

L Seminario 1.- Búsqueda de información financiera en Internet.

Seminario 2.- Base de datos SABI.

Seminario 3.- Lectura de la información de la Memoria de las Cuentas anuales de las empresas

Seminario 4.- A3CON: programa informático de contabilidad

Seminario 5.- Presentación de la información de las empresas.

4.- Ordene del 1 (máx.) al 5 (min.) los seminarios por orden de su percepción sobre el grado de dificultad.

Seminario 1.- Búsqueda de información financiera en Internet.

Seminario 2.- Base de datos SABI.

Seminario 3.- Lectura de la información de la Memoria de las Cuentas anuales de las empresas

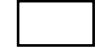

Seminario 4.- A3CON: programa informático de contabilidad

Seminario 5.- Presentación de la información de las empresas.

5.- Ordene del 1 (máx.) al 5 (min.) los seminarios por orden de su percepción sobre su utilidad.

Seminario 1.- Búsqueda de información financiera en Internet.

Seminario 2.- Base de datos SABI.

Seminario 3.- Lectura de la información de la Memoria de las Cuentas anuales de las empresas

Seminario 4.- A3CON: programa informático de contabilidad

Seminario 5.- Presentación de la información de las empresas. 
6.- ̇Con qué frase definiría mejor los seminarios recibidos? Elija solo una respuesta.

a) En los seminarios se analiza el contenido de la asignatura desde un enfoque práctico

b) En los seminarios se aprende a conocer la utilidad de los contenidos teóricos combinados con un enfoque dinámico y real.

c) Se trata de aumentar la carga de trabajo de la asignatura sin finalidad alguna.

d) En los seminarios no he aprendido nada interesante.

7.- Señale el principal inconveniente de los seminarios. Elija solo una respuesta:

a) La carga de trabajo de los seminarios es muy elevada.

b) La coordinación entre los aspectos teóricos de clase y los seminarios no ha sido buena.

c) No he recibido el feed-back de los seminarios.

d) No he encontrado inconvenientes a destacar.

8.- Comparando los seminarios de "contabilidad financiera" con otros seminarios recibidos,

a) los seminarios de contabilidad financiera han exigido más carga de trabajo que otros seminarios.

b) los seminarios de contabilidad financiera han sido más útiles que otros seminarios recibidos.

c) los seminarios de contabilidad financiera han sido más motivadores que otros seminarios recibidos.

d) los seminarios de contabilidad financiera han contribuido a profundizar mis conocimientos en contabilidad que en otras materias

9.- $\dot{\text { H}}$ a utilizado el Campus Virtual para los seminarios de contabilidad financiera?

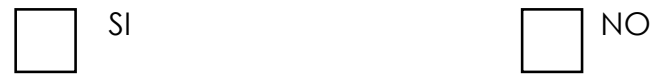

10.- ¿̇Cuál es el grado de satisfacción con el uso del campus virtual? (de 1 min al 10 max)

Los datos serán tratados de manera anónima. Muchas gracias por tu colaboración. 